\title{
An Ecn Approach to Congestion Control Mechanisms in Mobile Adhoc Networks
}

\author{
Som Kant Tiwari \\ Department of CSE \\ RITS, RGI,Group, \\ Bhopal,India
}

\author{
Dr.Y.K.Rana \\ Department of CSE \\ RITS, RGI,Group, \\ Bhopal,India
}

\author{
Prof. Anurag Jain \\ Department of CSE \\ RITS, RGI,Group, \\ Bhopal,India
}

\begin{abstract}
Node(s)/link(s) of a network are subjected to overloading; network performance deteriorates substantially due to network congestion. Network congestion can be mitigated with the help of Explicit Congestion notification (ECN) technique. ECN notification is carried out by setting ECN bit in the TCP header. This allows for end-to-end notification of network congestion without dropping packets. ECN bit notifies TCP sources of incipient congestion before losses occur. ECN is a binary indicator ( 1 bit) which does not reflect the congestion level completely and so its convergence speed is relatively low. In our work, we have used an extra ECN bit ( 2 bit ECN). The extra bit allows for passing of additional congestion feedback to the source node. This enables the source node to determine the level of congestion based on which steps can be taken to ensure faster convergence. In comparison to single bit ECN, the additional information afforded by double bit ECN allows for more flexibility to adjust window size, to handle congestion. Simulation results have shown that the proposed method improves overall performance of the network by over $12 \%$.
\end{abstract}

Keywords-Explicit Congestion Notification (ECN); Mobile ad hoc Networks (MANET); Congestion control; Congestion window Transmission Control Protocol (TCP)

\section{INTRODUCTION}

Mobile Ad hoc Network (MANET) is a distributed system of self-organizing and independent nodes. Mobility of nodes causes Network topology to change frequently. In the absence of infrastructure (due to its Ad-hoc characteristics) and dynamic topology, MANET nodes must self-reorganize themselves to forward each other's data to achieve end-to-end communication. As such, MANET nodes must not only behave as end nodes, but also as routers for forwarding packets. The ability to re-organize and self-operate makes MANET suitable for a number of applications requiring adhoc operation, including- for military and emergency scenarios. Self re-organization and self-operation requires adapting to variations in topology and bandwidth requirements, which is the main challenge in the design of MANET. Each node stores data to be forwarded in a buffer, until it is successfully forwarded. Frequent changes in bandwidth requirement (load) and topology can cause the buffer to get full as new packets arrive before older packets are forwarded. This results in newly arriving packets not finding space in the buffer, to wait for their turn to be forwarded; leading to them being dropped (loss).In an attempt to adapt to topology and traffic dynamics, additional control packet traffic is generated. This, in addition to the attempts to re-transmit lost packets, cause traffic surge at various nodes along the path. Nodes that is unable to cope with this surge form bottleneck. Bottleneck leads to congestion, which reduces the performance of whole network.

Mitigation of congestion (congestion control) is carried out at transport layer. In TCP (Transport Control Protocol) numbers of variants have been proposed for congestion control. They can be classified into two categories according to the mechanisms used to obtain congestion feedback: explicit network feedback based schemes and pure end-to-end schemes. Explicit network feedback based schemes usually perform better than pure end-to-end ones, but many of them require modifying IP header to carry additional feedback information. This incurs complicated computation in routers and makes them unpractical in real networks. For example, the $\mathrm{XCP}$ protocol can obtain accurate feedback, achieving high efficiency, fairness and fast convergence speed; but their additional feedback overhead also makes it difficult to deploy them in real networks. We propose an enhanced version of ECN scheme allows more flexible handling of congestion. This scheme is based on the network load factor $\rho_{l}$ which can be estimated very easily at each router and is associated with following illustrated ECN bits in the IP header for feedback. We are making the sender aware about the state of congestion of link in the network. This is done in our scheme through explicit feedback from the network in the form of explicit congestion notification (ECN). Depending on the feedback from the network, the sender act accordingly sudden increase/ decrease the congestion window. By using two ECN bits, it gives more convergence to adjust window size with added options to RED queue to handle congestion more effectively.

This paper is organized as follows: existing work described in section II, followed by proposed methodology is described in section III, simulation and result present in section IV, finally, we conclude this work in section V.

\section{EXISTING WORK}

The new queue management scheme is designed [8] to help the monitoring the global congestion situation of an autonomous system. In order to observe the congestion situation of the system, traffic is generated between routers and a centralized unit. Routers are send packets according to current output queue levels. The central unit monitors overall view of congestion and update their random early detection 
parameters according to the congestion notification of the control unit [8]. Strategical (RED) Random early detection method is proposed in [4] which use queue parameter. By monitoring the queue parameter according to current queue condition and reduces the queuing delay and increase the throughput. Delay time can be reduced substantially if network length is more and sender and receiver are at sufficient distance and increase the throughput. Exiting RED uses a mechanism early detection of Packet drop without waiting to queue overflow, this mechanism inform the sender to reduce the packet transmission rate and also inform the receiver to not to send excessive acknowledgement packets. In strategical RED parameters are varied according to current queue availability which send excessive acknowledgment and solve above problem.

In [2] this paper an ECN-based congestion control algorithm called access point congestion control (APCC) is presented. In this algorithm using both wireless channel load and buffer length as compound congestion indicator. It provides more stable and higher efficient TCP congestion control and achieve time fairness and higher total network goodput. [5] Also presented an ECN scheme with new response strategy that is more aggressive in the short term, but preserve TCP behavior in long term without modifying the router marking rate. An effort [7] is tried to present distributed ECN-based congestion control protocol to which we refer as Double-Packet Congestion Control Protocol (DPCP).This scheme provide more accurate feedback compare to variable structure congestion control protocol (VCP).By extracting the router information into series of packets .It is capable to notify three level of congestion in two ECN bits. In [9] explicit congestion notification accurately improves the efficiency of TCP without harming its performance. The Probabilistic Congestion Notification (PCN) is designed in [6] to help determine the exact level of congestion at each intermediate queue. This scheme use single bit explicit congestion notification (ECN) in the IP header. The source to estimate the exact level of congestion at each intermediate queue. By knowing this, the source mitigate its sending rate or choose an alternative roots. The estimation mechanism makes use of time series analysis both to improve the quality of the congestion estimation and to predict, ahead of time, the congestion level which subsequent packets will encounter [6].

[3] Have presented a new Code point mechanism that replace the current $\mathrm{ECN}$ mechanism and reuse the assigned bit in the TCP header. In order to observe the congestion situation and provide a better accuracy against losses of packets. This code point scheme performs well in internet link scenario and provides more accurate ECN feedback. By reusing the ECN TCP header bits without allocating further option space. In this paper [1], an innovative method variable structure congestion control with bandwidth estimation (VCP-BE) is presented. That uses available end-to-end bandwidth estimation to provide more accurate congestion feedback. With estimated available bandwidth and ECN feedback window adjustment, the algorithm convergence fast and to improve the network performance. But this algorithm suffers from overestimation of bandwidth and difficult computation in routers.

\section{PROPOSED APPROACH}

In this work enhanced explicit congestion notification technique is used. The proposed enhanced version employs extra bit to provide additional traffic information at the senders end. This additional information allows more flexible handling of congestion. The additional traffic information carried in the extra ECN bit, allows the node to determine more suitable adjustments to the congestion window size, with added options to RED queue to handle congestion more effectively. Conventionally, size of sliding window is decided upon successful delivery acknowledgement of packets and it gradually increases and decreases accordingly. In earlier works, RED queue was administered by two values of minimum and maximum threshold value, but here in this work RED queue is managed by introducing two more threshold value which are depicted by slow decrement and fast decrement along with similar vice versa pattern, due to highly dynamic nature of MANETs.

The network state is based on the network load factor $\rho_{l}$ which can be estimated very easily at each router and is associated with following illustrated ECN bits in the IP header of each data packet. In our approach, for every time interval $t_{p}$ a router observes its input traffic and calculates the load factor $\rho_{l}$ as-

$$
\rho_{l}=\frac{\lambda+k . q}{\gamma \cdot C . t_{p}}
$$

Where $\lambda$ and $\mathrm{q}$ represent the amount of input traffic and the persistent queue length during the period $t_{p}, \mathrm{k}$ controls how quickly the persistent queue length can be reduced to zero. $\gamma$ Denotes the target utilization and $\mathrm{C}$ indicates the channel capacity.

To adjust size of sliding window, an IMP will maintain a RED queue with four threshold level i.e. min, slow min, slow max, max which when get overburdened and crosses the max threshold level, set both ECN bits which signifies to reduce the window size significantly (let's say, four times) and if RED queue signals slow max threshold level, it reduces the size of window but less than aforesaid case (let's say two times). Below illustrated table expounds the behavior of RED queue which leads to change in ECN bits and adjustment in window size to handle congestion.

Following the aforesaid approach, as and when congestion experienced, the sliding window size is accordingly handled by the control bits sent by intermediate router to the source node. The novelty of the proposed approach lies in using additional traffic information, carried in extra ECN bit, to adjust window size more appropriately, instead of drastically slashing it down. As we are using the Mobile ad hoc network platform which is highly dynamic in nature and connectivity of any node changes abruptly, which sometimes leads to sudden increase/decrease of window size, but sometimes the same is needed to lesser extent, and such situation is handled in a better way in the proposed approach. 
TABLE 4.1. BEHAVIOR OF RED QUEUE AND ECN BITS

\begin{tabular}{|l|l|l|}
\hline RED queue threshold level & $\begin{array}{c}\text { ECN } \\
\text { bits } \\
\text { status }\end{array}$ & $\begin{array}{l}\text { Action to be taken } \\
\text { at senders side }\end{array}$ \\
\hline slow $\left(0 \leq \rho_{l} \leq 0.7\right)$ & 00 & $\begin{array}{l}\text { Increase window } \\
\text { size significantly } \\
(4 \text { times })\end{array}$ \\
\hline slow $\min \left(0.7 \leq \rho_{l} \leq 0.9\right)$ & 01 & $\begin{array}{l}\text { Increase window } \\
\text { size gradually } \\
(2 \text { times })\end{array}$ \\
\hline slow max $\left(0.9 \leq \rho_{l} \leq 1.0\right)$ & 10 & $\begin{array}{l}\text { Decrease window } \\
\text { size gradually } \\
(2 \text { times })\end{array}$ \\
\hline max $\left(\rho_{l}>1.0\right)$ & 11 & $\begin{array}{l}\text { Decrease window } \\
\text { size significantly } \\
(4 \text { times })\end{array}$ \\
\hline
\end{tabular}

\section{SimULATION AND RESULTS}

To evaluate the effectiveness of the proposed approach, aforesaid concept has been implemented over a simulation environment using NS2 simulator. In the simulations, we use the $400 * 400 \mathrm{~m}^{2}$ gride and set the data packet size as $512 \mathrm{~B}$. The basic setting is an $11 \mathrm{Mbps}$ link with a $80 \mathrm{~ms}$ RTT where the forward and reverse path each has 5FTP flows unless stated otherwise. RED is always used with ECN enabled at the routers. All simulations are run for at least 120s. The effectiveness of the approach is being justified by comparing our approach with drop tail approach in literature work illustrated on the basis of Goodput, which is the application level throughput, i.e. the number of useful information bits delivered by the network to a certain destination per unit of time. The amount of data considered excludes protocol overhead bits as well as retransmitted data packets. This is related to the amount of time from the first bit of the first packet sent (or delivered) until the last bit of the last packet is delivered.

Another metrics is average queue length which is a crucial count to measure congestion. A low value of average queue length a better congestion control mechanism is being used.Lastly, loss rate is being considered for the same which is defined as the successfully delivered packets to the total sent packets.

Following mentioned graphs illustrate the effectiveness of our approach as compare to its counterpart drop tail.

From figure 5.1, it is clearly depicted that our approach named "MyRED" is performing better consistently over increasing number of flows.

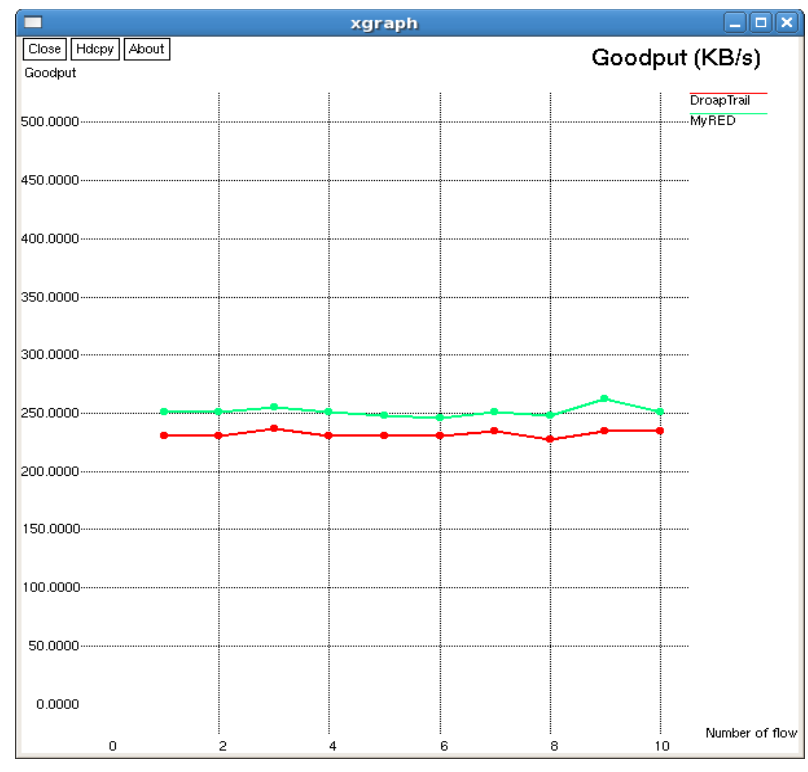

Fig 5.1. Goodput comparison of proposed approach

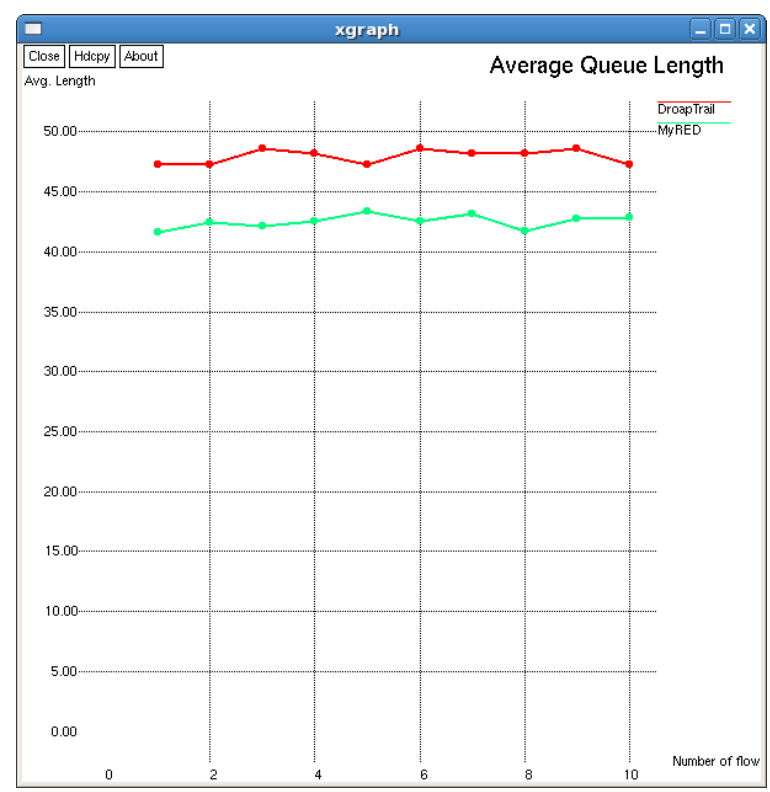

Fig 5.2. Average queue length comparison of proposed approach

Figure 5.2 expounds comparison of average queue length between proposed approach v/s drop tail and here also, our proposed approach MyRED is showing better results than its counterpart. Although it is fluctuating too much but still it is too low when compared to Drop tail approach. 


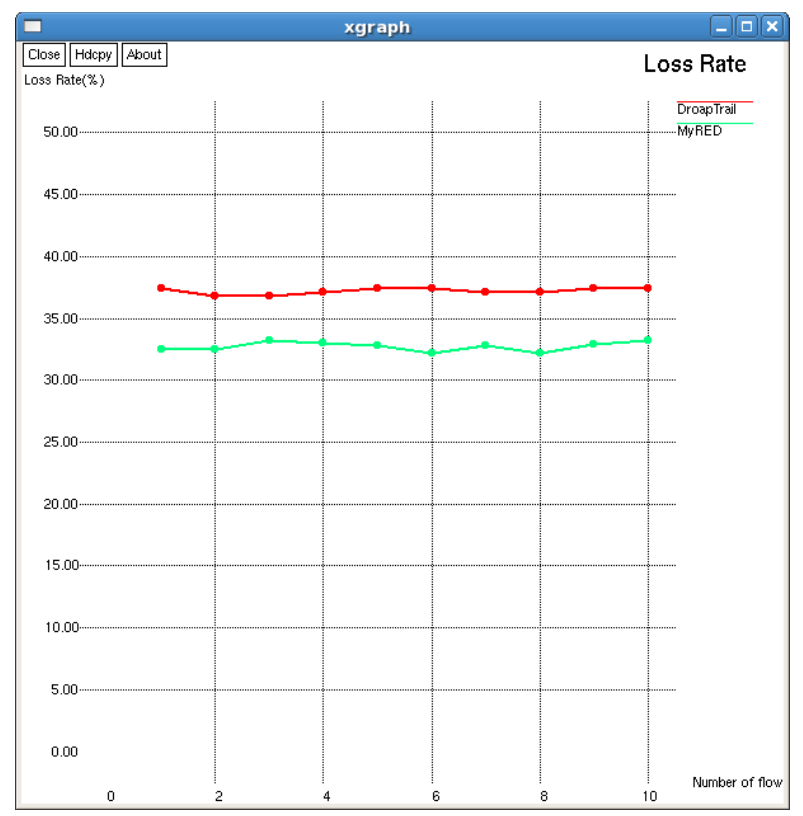

Fig 5.3. Loss rate comparison of proposed approach

Lastly the loss rate observed in simulation setup is also far better (lesser) in our approach in comparison to that of Drop tail approach.

\section{CONCLUSIONS}

In the aforesaid study and evaluation of approach, we found our approach more effective and suited for congestion prone networks to handle the same using ECN bits associated with load factor. In all above expounded parameters, MyRED approach is performing better than its variant. The work can be extended and enhanced by implementing it in real hardware to study its strength and flaws in more practical settings.

\section{REFERENCES}

[1] Jianxin Wang, Jie Chen, Shigeng,Weiping Wang, "An explicit congestion control protocol based on bandwidth estimation"978-1-42449268-8/11/\$26.00,IEEE,2011.

[2] Jiawei Huang, Jinaxin Wang, "An ECN-Based Congestion Control Algorithm for TCP Enhancement in WLAN"11th IEEE International Conference on High Performance Computing and Communications, 2009.

[3] Mirja K"uhlewind, Richard Scheffenegger," Design and Evaluation of Schemes for More Accurate ECN Feedback" Workshop on Telecommunications 2012.

[4] Jeetendra $\mathrm{Kr}$ Patel, Jigyasu Dubey, "Mobile Ad hoc Network Performance ImprovementUsing Strategical RED" 978-1-4673-19898/12/\$31.00, IEEE, 2012.

[5] Minseok Kwon and Sonia Fahmy," TCP Increase/Decrease Behavior with Explicit Congestion Notification" 0-7803-7400-2/02/\$17.00 () 2002 IEEE.

[6] Mussie Woldeselassie, Richard G. Clegg, Miguel Rio," Forecasting Full-Path Network Congestion Using One Bit Signalling" 978-1-42446404-3/10/\$26.00 @2010 IEEE.

[7] Xiaolong Li,Homayoun Yousefi Zadeh, "Distributed ECN-based congestion control" 978-1-4244-3435-0/09/\$25.00, IEEE,2009.

[8] Ozen Yelbasi, Emin German, "A New Approach to Estimate RED Parameters Using Global Congestion Notification", International Conference on Network Computing and Information Security, 2011.

[9] Stefanos Harhalakis, Nikolaos Samaras, Vasileios Vitsas" An experimental study of the efficiency of Explicit Congestion Notification" 978-0-7695-4389-5/11 \$26.00 @ 2011 IEEE. 